\title{
Toward a New Axiomatic for Hyper-connections
}

\author{
Benjamin Perret ${ }^{1}$, Sébastien Lefèvre ${ }^{2}$, and Christophe Collet $^{1}$ \\ 1 Image Science, Computer Science and Remote Sensing Laboratory (LSIIT, UMR \\ 7005, University of Strasbourg-CNRS). Address: LSIIT, Pôle API, Bd Sébastien \\ Brant, BP 10413, 67412 Illkirch Cedex France \{bperret,c.collet\}@unistra.fr \\ 2 VALORIA Laboratory, University of South Brittany. Address: VALORIA Lab, \\ Campus de Tohannic, BP 573, 56017 Vannes Cedex, France \\ sebastien.lefevre@univ-ubs.fr
}

\begin{abstract}
We propose an evolution of the hyper-connection axiomatic in order to improve the consistency of hyper-connected filters and to simplify their design. Our idea relies on the principle that the decomposition of an image into h-components must be necessary and sufficient. We propose a set of three equivalent axioms to achieve this goal. We show that an existing h-connection already fulfills these axioms and we propose a new h-connection based on flat functions that also fulfills these axioms. Finally we show that these new axioms bring several new interesting properties that simplify the use of h-connections and guarantee the consistency of h-connected filters as they ensure that: 1) every deletion of image components will effectively modify the filtered image 2) a deleted component can not re-appear in the filtered image.
\end{abstract}

\section{Introduction}

Connections in image processing describe how pixels can be grouped together according to their spatial relationships and/or their gray level values. In recent years, several works were devoted to the development of new theories of connections among which hyper-connection (h-connection) $[1-3]$ is a very promising notion. Contrary to traditional connections (like set connections), h-connections allow to decompose images into intersecting components.

In this paper, we investigate the problem of the consistency of connected filters (edge-preserving filters) based on h-connections. The usual way to produce a h-connected filter on an image is based on the following three steps: 1) consider the set of h-connected components of this image, 2) select a subset of theses components and 3) reconstruct an image using the select h-components. In this scheme, one expect that the result image contains the selected h-components and only those ones. But in the current theory, we can easily show that the result will contain the selected h-connected components but may also contain other components. These unwanted components are in fact h-components of the original image that have not been selected during the $2^{\text {nd }}$ step (see proposition 2 further). This undesirable effect can be circumvented by the addition of a new property that relies on the idea that the decomposition of an image into h-components 
must be necessary and sufficient to describe the image. We think that this property is fundamental for image processing as it implies that the decomposition into h-components completely describes the whole image (sufficient) and that none of its components is useless (necessary). These requirements enforce the consistency of the h-connected filters as they ensure that: 1) every deletion of image components will effectively modify the filtered image 2) a deleted component can not re-appear in the filtered image. From a more formal point of view, the sufficiency and necessity conditions can be formulated like this: the supremum of the h-components of an image must be equal to the whole image (sufficient) and the supremum of a family strictly included in the family of h-components must be strictly included in the image (necessary).

In this article we propose a set of three axioms to achieve this goal and we show that they are indeed equivalent. We show that an existing h-connection (the functions with a unique maximum) already fulfills these axioms and we propose a new h-connection based on flat functions, that may be seen as a generalization of the flat zones, that also fulfills these axioms. Finally we show that these new axioms bring several new interesting properties that simplify the use of h-connections and guarantee the consistency of h-connected filters.

This article is organized as follows: section 2 gives some preliminary definitions, section 3 presents the theory of h-connections and establishes some new results, section 4 presents our new theoretical developments and finally section 5 concludes this work.

\section{Lattice and Set Connection}

This section gives some preliminary definitions about lattices and set connections. In the sequel, sets and families are written in capital letters while elements of a set are written in lower-case letters. The logical conjunction (respectively disjunction) is noted $\wedge$ (respectively $\vee$ ).

A lattice $(\mathcal{L}, \leq, \bigvee, \wedge)$ is composed of a non-empty set $\mathcal{L}$ with a partial order $\leq$ and two operators: a supremum $\bigvee$ and an infimum $\Lambda$. In the following, we consider only complete lattices where every non empty family $A \subseteq \mathcal{L}$ has a supremum $\bigvee A=\top$ and an infimum $\bigwedge A=\perp$ in $\mathcal{L}$. A set $\mathcal{S} \subseteq \mathcal{L}$ such that $\perp \notin \mathcal{S}$ is called a sup-generating family of $\mathcal{L}$ if every element of $\mathcal{L}$ can be written as the supremum of elements of $\mathcal{S}(\forall a \in \mathcal{L}, \exists B \subseteq \mathcal{S}, a=\bigvee B)$. The elements of $\mathcal{S}$ are called sup-generators. Important examples of lattices are:

- the extended real line $\overline{\mathbb{R}}=\mathbb{R} \bigcup\{-\infty,+\infty\}$, which is a complete chain under the usual order, infimum, and supremum. It is sup-generated by $\mathbb{R}$.

- the set of all subsets of a set $E$ (noted $\mathcal{P}(E)$ ), which is a complete lattice with the partial order defined by the inclusion relation, and the infimum and supremum given by the set intersection and union. It is sup-generated by the singletons of $E$.

- the set of functions from a set $E$ into a lattice $\mathcal{L}$ (noted $\mathcal{L}^{E}$ ), which is a lattice under the pointwise order $\forall f, g \in \mathcal{L}^{E}, f \leq g \Leftrightarrow \forall x \in E, f(x) \leq_{\mathcal{L}} g(x)$. The 
infimum and supremum are similarly defined by a pointwise application of the infimum $\bigwedge_{\mathcal{L}}$ and supremum $\bigvee_{\mathcal{L}}$ of the underlying lattice. $\mathcal{L}^{E}$ is supgenerated by the pulses: the functions $\delta_{x}^{t}, \forall x \in E, \forall t \in \mathcal{L}, t \neq \perp$ defined by $\forall y \in E, \delta_{x}^{t}(y)=t$ if $x=y, \perp$ otherwise. The properties of $\mathcal{L}^{E}$ depend of the properties of the underlying lattice $\mathcal{L}$.

A connection on $\mathcal{P}(E)$ is a family $\mathcal{C} \subseteq \mathcal{P}(E)$ composed of the connected elements of $E$. Formally we say that $\mathcal{C}$ is a connection if [4]:

1. $\{\emptyset\} \in \mathcal{C}$ : the empty set is connected;

2. $\forall x \in E,\{x\} \in \mathcal{C}$ : the singletons (or points) are connected;

3. $\forall A \subseteq \mathcal{C}, \bigcap A \neq \emptyset \Rightarrow \bigcup A \in \mathcal{C}$, the union of intersecting connected elements is connected.

\section{Hyper-connection}

We now present the theory of h-connections, we give the definition of a new h-connection and we establish two new properties. Being given a lattice $\mathcal{L}$ with a sup-generating family $\mathcal{S}$, an h-connection $\mathcal{C}^{+}$on $\mathcal{L}$ is a subset of $\mathcal{L}$ verifying the following conditions [1]:

1. $\perp \in \mathcal{C}^{+}$: the least element is h-connected;

2. $\forall s \in \mathcal{S}, s \in \mathcal{C}^{+}$: the points (i.e. the sup-generators) are h-connected;

3. $\forall A \subseteq \mathcal{C}, \bowtie A \Rightarrow \bigvee A \in \mathcal{C}^{+}$, the supremum of overlapping h-connected elements is h-connected.

where $\bowtie$ is a predicate on $\mathcal{P}(\mathcal{L})$ called the overlap criterion. This predicate must be decreasing: $\forall A \subseteq \mathcal{P}(\mathcal{L}), \bowtie A \Rightarrow \forall b \in \mathcal{L}, \bowtie\{b\} \bigcup A$.

A simple example of h-connection is made of functions with a unique maximum $[1,2]$. This h-connection is defined on the lattice of functions $\mathcal{L}^{E}$ and it is based on a primary set connection $\mathcal{C}_{o}$ on $E$. Then, one says that a function has a unique maximum if it is connected at all levels with respect to the primary set connections. More formally, we define the set of connected functions by: $\mathcal{C}_{m}^{+}=\left\{f \in \mathcal{L}^{E} \mid \forall t \in \mathcal{L}, \bar{f}^{t} \in \mathcal{C}_{o}\right\}$, with $\vec{f}^{t}=\{p \in E \mid t \leq f(p)\}$ the thresholding of $f$ at level $t$. The overlap criterion can be defined as: $\forall\left\{f_{i}\right\} \subseteq \mathcal{L}^{E}, \bowtie_{m}\left\{f_{i}\right\} \Leftrightarrow$ $\forall t \in \mathcal{L}, U_{t}=\emptyset$ or $\bigcap U_{t} \neq \emptyset$ with $U_{t}=\left\{{\overrightarrow{f_{i}}}^{t} \mid{\overrightarrow{f_{i}}}^{t} \neq \emptyset\right\}$.

Another new example of h-connection is given by the set of all flat functions. Let $\mathcal{L}^{E}$ be the image space, let $\mathcal{C}_{o}$ be a primary set connection on $\mathcal{P}(E)$, the set of flat functions is defined by:

$$
\mathcal{C}_{p}^{+}=\left\{f_{C, t} \in \mathcal{L}^{E} \mid C \in \mathcal{C}_{o}, t \in \mathcal{L}\right\}
$$

with $\forall x \in E, f_{C, t}(x)=t$ if $x \in C, \perp$ otherwise. Then the overlap criterion $\bowtie_{p}$ is defined by:

$\forall\left\{g_{i \in I}\right\} \subseteq \mathcal{C}_{p}^{+}, \bowtie_{p}\left(\left\{g_{i}\right\}\right)= \begin{cases}\text { true } & \text { if } U \neq \emptyset \text { and } \forall i, j \in I, \forall x \in U, g_{i}(x)=g_{j}(x) \\ \text { false } & \text { otherwise }\end{cases}$ 
with $U=\bigcap_{i \in I} \operatorname{supp}\left(g_{i}\right)$ and $\operatorname{supp}\left(g_{i}\right)=\left\{p \in E \mid g_{i}(p) \neq \perp\right\}$ is the support of the function $g_{i}$. Flat functions are overlapping if their supports intersect and if they have the same value on this intersection. Figure 1 shows examples of overlapping and non-overlapping flat functions according to $\bowtie_{p}$. Contrary to the usual definition of flat zones (the largest connected component such that the function is constant), the h-connected flat zones can spread under other flat zones that have a higher level. Several flat zones according to the usual definition can then be represented by a unique h-connected flat function.

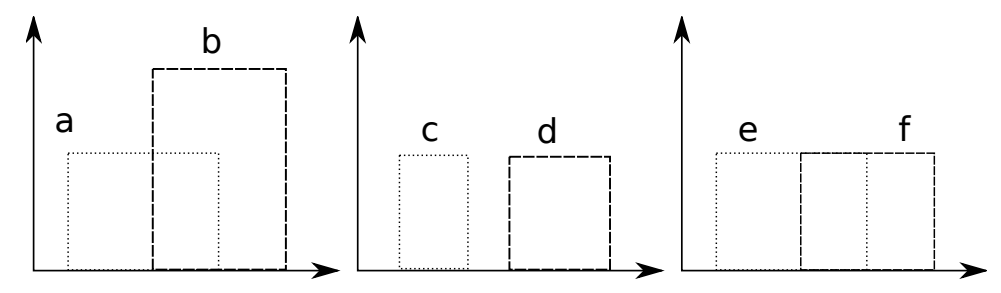

Fig. 1. The overlap criterion $\bowtie_{p}$. Functions a and b are not overlapping because they don't have the same value on their intersection. Functions $\mathrm{c}$ and $\mathrm{d}$ are not overlapping because their supports do not intersect. Functions e and f overlap.

Proposition 1. $\left(\mathcal{C}_{p}^{+}, \bowtie_{p}\right)$ is an h-connection of $\mathcal{L}^{E}$ (see Figure 2).

Proof. We must show that the three axioms of the h-connection are verified by $\left(\mathcal{C}_{p}^{+}, \bowtie_{p}\right)$. First note that $\bowtie_{p}$ is trivially decreasing.

1) By definition of set connections, $\emptyset \in \mathcal{C}_{o}, \forall t \in \mathcal{L}, f_{\emptyset, t}=\perp \in \mathcal{C}_{p}^{+}$.

2) By definition of set connections, the singletons of $E$ belong to $\mathcal{C}_{o}$, thus, $\forall x \in E, \forall t \in \mathcal{L}$, the $f_{\{x\}, t}=\delta_{x, t} \in \mathcal{C}_{p}^{+}$are the pulses of $\mathcal{L}^{E}$ which are a sup-generating family $\mathcal{S}$ of $\mathcal{L}^{E}$.

3) Let $\left\{a_{i \in I}\right\}$ be a family of $\mathcal{C}_{p}^{+}$, let also $U=\bigcap_{i \in I} \operatorname{supp}\left(a_{i}\right)$, and assume that $U \neq \emptyset$ and $\forall i, j \in I, \forall x \in U, a_{i}(x)=a_{j}(x)$ then we must show that $\bigvee a_{i} \in \mathcal{C}_{p}^{+}$. From property 3$)$ of set connections, if $U \neq \emptyset$ then $\bigcup_{i \in I} \operatorname{supp}\left(a_{i}\right)=R \in \mathcal{C}_{o}$, then from the definition of $\mathcal{C}_{p}^{+}$and from the condition $\forall i, j \in I, \forall x \in U, a_{i}(x)=a_{j}(x)$, all $a_{i}$ have the same value $t$ on their supports, thus, $\bigvee a_{i}=f_{R, t} \in \mathcal{C}_{p}^{+}$.

Hyper-connected operators and openings H-operators [3] are applications from $\mathcal{L}$ into $\mathcal{P}(\mathcal{L})$ which extract the h-components marked by a sup-generator. Formally, being given a sup-generator $s \in \mathcal{S}$, we define the h-operator by:

$$
\forall a \in \mathcal{L}, \quad \gamma_{s}^{*}(a)=\left\{h \in \mathcal{C}^{+} \mid s \leq h \leq a, \forall g \in \mathcal{C}^{+}, h \leq g \leq a \Rightarrow g=h\right\}
$$

The h-components are maximal h-connected elements and the h-components of $a \in \mathcal{L}$ are given by:

$$
\gamma^{*}(a)=\bigcup_{s \in \mathcal{S}} \gamma_{s}^{*}(a)
$$


The h-opening marked by $s$ is the supremum of the h-components of $a$ above $s$ :

$$
\forall a \in \mathcal{L}, \quad \gamma_{s}(a)=\bigvee \gamma_{s}^{*}(a)
$$

The two following properties hold [2]:

1. $a=\bigvee \gamma^{*}(a)$ : an element is the supremum of its h-components;

2. $\forall b, c \in \gamma^{*}(a), b \neq c \Rightarrow b \bowtie c$ : two h-components do not overlap.

Z-operators In [2], the authors propose the interesting notion of $z$-operators. Let $a \in \mathcal{L}$, the equivalence relation $\stackrel{a}{\sim}$ on $\mathcal{S}$ is defined by:

$$
\forall b, c \in \mathcal{S}, \quad b \stackrel{a}{\sim} c \Leftrightarrow \gamma_{b}(a)=\gamma_{c}(a)
$$

Then, the z-operator is defined as the supremum of an equivalence class of $\stackrel{a}{\sim}$ :

$$
\forall s \in \mathcal{S}, \quad \zeta_{s}(a)=\bigvee\{b \in \mathcal{S} \mid b \stackrel{a}{\sim} s\}
$$

Finally, the set of all z-zones of $a$ is noted:

$$
\zeta(a)=\left\{\zeta_{s}(a) \mid \forall s \in \mathcal{S}\right\}
$$

The authors of [2] have chosen to base the z-operators on the h-connected openings, but we can also define them in terms of h-connected operators leading to a slightly different definition. Let $\stackrel{a_{*}}{\sim}$ be the equivalence relation defined by:

$$
\forall b, c \in \mathcal{S}, \quad b \stackrel{a_{*}^{*}}{\sim} c \Leftrightarrow \gamma_{b}^{*}(a)=\gamma_{c}^{*}(a)
$$

The alternative z-operator* is the supremum of an equivalence class of $\stackrel{a}{\sim} *^{*}$ :

$$
\forall s \in \mathcal{S}, \quad \zeta_{s}^{*}(a)=\bigvee\left\{b \in \mathcal{S} \mid b \stackrel{a_{*}^{*}}{\sim} s\right\}
$$

Generally, as h-components can be intersecting, $\zeta_{s}(a) \neq \zeta_{s}^{*}(a)$. Consider the set $E=\{a, b, c\}$ and the h-connection $\{\emptyset,\{a\},\{b\},\{c\},\{a b\},\{b c\},\{a c\}\}$ on $\mathcal{P}(E)$ with the overlap criterion defined as false (two elements are never overlapping, this is a valid criterion since it is trivially decreasing). Then, the h-components of $E$ are $\{\{a b\},\{b c\},\{a c\}\}$. Thus, we have $\gamma_{a}(E)=\gamma_{b}(E)=\gamma_{c}(E)=E$ and $\zeta_{a}(E)=\zeta_{b}(E)=\zeta_{c}(E)=E$. But $\gamma_{a}^{*}(E)=\{\{a b\},\{a c\}\}, \gamma_{b}^{*}(E)=\{\{a b\},\{b c\}\}$ and $\gamma_{c}^{*}(E)=\{\{a c\},\{b c\}\}$ thus $\zeta_{a}^{*}(E)=\{a\} \neq \zeta_{a}(E)$. In section 4, we show that under certain conditions, we have $\forall a \in \mathcal{L}, \forall s \in \mathcal{S}, \zeta_{s}(a)=\zeta_{s}^{*}(a)$. Figure 2 shows an example of function decomposition with the z-operators and the $\mathrm{h}$-connection of functions having a unique maximum.

H-reconstruction Being given a marker $m \in \mathcal{L}$, the hyper-reconstruction (hreconstruction) of $a \in \mathcal{L}$ marked by $m$ is defined by [2]:

$$
\lceil a\rceil_{m}=\bigvee_{s \in \mathcal{S}, s \leq m} \gamma_{s}(a)
$$




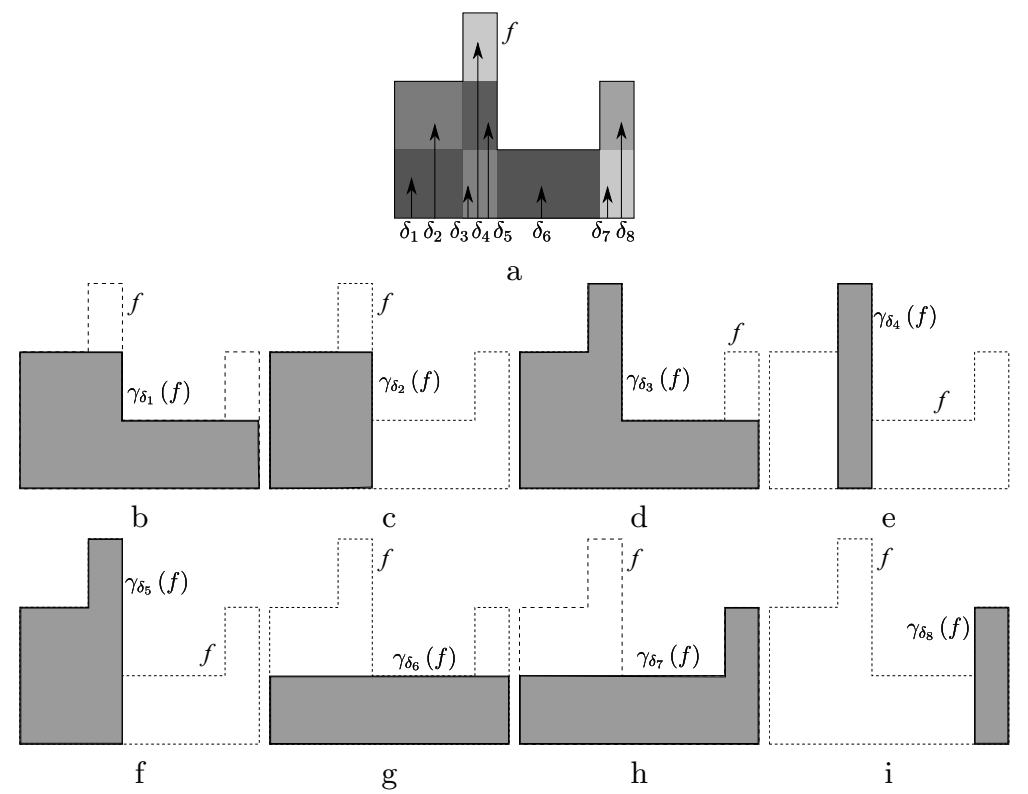

Fig. 2. Example of decomposition of the function $f$ using the h-connection of flat functions. Figure (a) shows the function $f, 8$ pulses $\delta_{1}, \ldots, \delta_{8}$ that are representative of the 8 equivalence classes of the sup-generating family according to $\stackrel{f}{\sim}$. In image (a), each zone of $f$ marked by the pulses $\delta_{i}$, corresponds to an equivalence class and is thus the result of a z-operator $\zeta_{\delta_{i}}(f)$. Figures (b) to (i) show the results of the h-openings of $f$ marked by the $\delta_{i}$. One can note that figures (c), (e), (g) and (i) are the h-components of $f$ while the results of the h-opening marked by $\delta_{1}, \delta_{3}, \delta_{5}$ and $\delta_{7}$ represented in figures (b), (d), (f) and (h) are not h-connected.

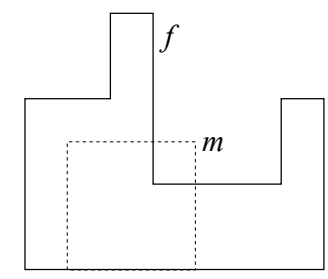

a

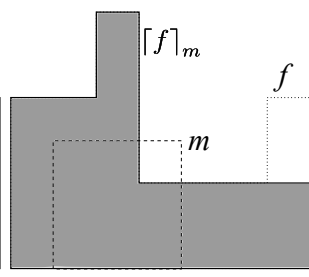

$\mathrm{b}$

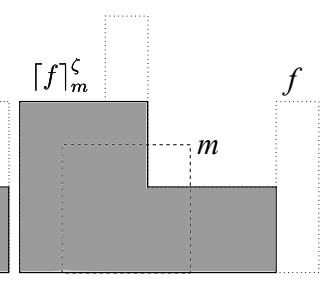

c

Fig. 3. Example of h-reconstruction with the h-connection of flat functions. Figure (a) shows the function $f$ and the marker $m$. The figure (b) represents the result of the h-reconstruction $\lceil f\rceil_{m}$ of $f$ by $m$ proposed in [2]. Figure (c) represents the result $\lceil f\rceil_{m}^{\zeta}$ of $f$ by $m$ with our definition. 
We propose another definition based on the alternative z-operators:

$$
\lceil a\rceil_{m}^{\zeta}=\bigvee_{s \in \mathcal{S}, s \leq m} \zeta_{s}(a)
$$

The latter is more flexible, the two approaches are compared in Figure 3.

New properties We establish here two properties that will help us in following proofs. Let $a \in \mathcal{L}$ :

Proposition 2. $\forall\left\{h_{i}\right\} \subseteq \gamma^{*}(a),\left\{h_{i}\right\} \subseteq \gamma^{*}\left(\bigvee\left\{h_{i}\right\}\right)$

Proof. Let $a$ in $\mathcal{L},\left\{h_{i}\right\} \subseteq \gamma^{*}(a)$ and $b=\bigvee\left\{h_{i}\right\}$, for all $i$, we have $h_{i} \leq b$ and $b \leq a$. Suppose that there exists some $h \in \mathcal{C}^{+}$such that $h_{i} \leq h \leq b \leq a$. But by definition of $\gamma^{*}(a)$ and as $h_{i}$ belongs to $\gamma^{*}(a)$, we have $h_{i}=h$ showing that $h_{i} \in \gamma^{*}(b)$. Thus we have $\left\{h_{i}\right\} \subseteq \gamma^{*}\left(\bigvee\left\{h_{i}\right\}\right)$.

This property that was already known under a different form in [2] states that: being given a family of h-components, the h-components of its supremum is a superset of the given family. Those two families are generally not equal, take for example the h-connection given in section 3 ; the h-components of the element $\{a b c\}$ are $\{\{a b\},\{b c\},\{a c\}\}$. Now, consider the subfamily $\{\{a b\},\{b c\}\}$, the supremum of this family is equal to $\{a b c\}$ and the h-components of this supremum is a strict superset of the family.

Proposition 3. $\forall s \in \mathcal{S}, \forall h^{\prime} \in \gamma^{*}(a)$, if $s \leq h^{\prime}$ and $\gamma_{s}(a)=h \in \mathcal{C}^{+}$then $h=h^{\prime}$.

Proof. Let $s$ in $\mathcal{S}, h \in \mathcal{C}^{+}$such that $\gamma_{s}(a)=h$ and $h^{\prime} \in \gamma^{*}(a)$ such that $s \leq h^{\prime}$. We have $h^{\prime} \leq \bigvee\left\{g \in \gamma^{*}(a) \mid s \leq g\right\}=h$, so $s \leq h^{\prime} \leq h \leq a$ and by definition of h-components $h=h^{\prime}$.

If the h-opening of $a$ marked by the sup-generator $s$ is an h-component then there is no other h-component of $a$ above $s$.

In the following, we assume the existence of a complete lattice $\mathcal{L}$ with the sup-generating family $\mathcal{S}$ and the h-connection $\mathcal{C}^{+}$such that the number of hcomponents of each element of $\mathcal{L}$ is finite.

\section{Toward a new axiomatic}

Despite the successful developments of h-connections in recent works $[3,5-8$, $2]$, the theory of h-connections is still not satisfactory. All of the successes obtained have been based on specific h-connections, whereas only a few general properties have been established. This lack of theoretical results comes from the very broad definition of h-connections. The third axiom (the overlap criterion) is especially problematic. On one hand, this axiom is satisfactory because it formalizes the intuitive union based approach. On the other hand, the definition of 
the overlap criterion is so wide that, in practice, this third axiom does not bring any meaningful property to the h-connection (for example, consider the overlap criterion which is always false, and the third axiom is never applicable). One can also observe that in all the h-connections used in practice $[1,2,7,5,6]$ the overlap criterion is defined as a tautology such that it correctly fits the set of connected elements. For example, instead of a constantly false overlap criterion, one can also define the following less trivial valid overlap tautologic criterion: $\forall \mathcal{C}^{+} \in \mathcal{L}, \forall A \subseteq \mathcal{C}^{+}, \bowtie A \Leftrightarrow\left(\forall B \subseteq A, \bigvee B \in \mathcal{C}^{+}\right)$

To harden the definition of h-connections, we propose the fundamental property that an h-connection must provide for each element a decomposition in h-components that is necessary and sufficient. With the current theory it is clear that the decomposition in h-components is sufficient in the sense that an element is equal to the supremum of its h-components. But in general this decomposition is not necessary in the sense that only a subset of the h-components of an element can be necessary to retrieve the element by supremum.

More formally, one can define the following properties $\forall a \in \mathcal{L}$ :

P-1 - $\forall h^{\prime} \in \gamma^{*}(a), \bigvee\left\{h \in \gamma^{*}(a) \mid h \neq h^{\prime}\right\}<\bigvee \gamma^{*}(a)$ : all h-components of an element are necessary to describe the element.

P-2 - $\forall\left\{h_{i}\right\} \subseteq \gamma^{*}(a), \forall h \in \gamma^{*}(a), h \leq \bigvee\left\{h_{i}\right\} \Rightarrow h \in\left\{h_{i}\right\}$ : an h-component cannot be covered by other h-components.

P-3 - $\forall h \in \gamma^{*}(a), \exists s \in \mathcal{S}, \gamma_{s}^{*}(a)=h$ : each h-component of an element can be individually retrieved through an h-opening.

Then, the following proposition holds:

Proposition 4. Properties P-1, P-2, and P-3 are equivalent. In the following, we will say that a connection is accessible if the previous properties hold.

Proof. We first show P-2 $\Rightarrow$ P-1. Let $\left\{h_{i}\right\} \subseteq \gamma^{*}(a)$, and assume that $\exists h^{\prime} \in\left\{h_{i}\right\}$ such that $\bigvee\left\{h \in \gamma^{*}(a) \mid h \neq h^{\prime}\right\}=\bigvee \gamma^{*}(a)$. Let $F=\bigvee\left\{h \in \gamma^{*}(a) \mid h \neq h^{\prime}\right\}$, one have $h^{\prime} \leq \bigvee \gamma^{*}(a)=\bigvee F$ but that contradicts P-2 since $h^{\prime} \notin F$.

Then, we show $\mathrm{P}-1 \Rightarrow \mathrm{P}-2$ : let $\left\{h_{i}\right\} \subset \gamma^{*}(a)$ and assume that there exists $h \in \gamma^{*}(a)$ such that $h \notin\left\{h_{i}\right\}$ and $h \leq \bigvee\left\{h_{i}\right\}$. Let $b=\bigvee\left\{h_{i}\right\}=\bigvee\left(\left\{h_{i}\right\} \cup\{h\}\right)$, we have by proposition 2 that $h \in \gamma^{*}(b)$ and that $\left\{h_{i}\right\} \subseteq \gamma^{*}(b)$. And then we have $\bigvee\left\{h^{\prime} \in \gamma^{*}(b) \mid h^{\prime} \neq h\right\}=\bigvee\left\{h_{i}\right\}=b$ which contradicts P-1.

Now P-3 $\Rightarrow \mathrm{P}-2$ : let $\left\{h_{i}\right\} \subseteq \gamma^{*}(a)$ and $h \in \gamma^{*}(a)$. Assume that $h \leq \bigvee\left\{h_{i}\right\}$ then, by P-3, there exists $s \in \mathcal{S}$ such that $\gamma_{s}(a)=h$ and $s \leq h \leq \bigvee\left\{h_{i}\right\}$. By assumption, the family $\gamma^{*}(a)$ is finite, so there exists $j$ such that $s \leq h_{j}$ and from proposition 3 we can say that $h=h_{j}$ and thus $h$ belongs to $\left\{h_{i}\right\}$.

And finally, P-1 $\Rightarrow \mathrm{P}-3$ : let $h \in \gamma^{*}(a)$, P-1 implies that $\bigvee B<a$ with $B=$ $\left\{h^{\prime} \in \gamma^{*}(a) \mid h^{\prime} \neq h\right\}$. Now, consider the smallest family $\left\{s_{i}\right\} \subseteq \mathcal{S}$ such that $\bigvee\left(B \cup\left\{s_{i}\right\}\right)=a$. Let $s$ be an element of $\left\{s_{i}\right\}$, we have for all $h^{\prime} \in B, s \not \leq h^{\prime}$ and $s \leq h$ thus $\gamma_{s}(a)=h$.

P-1 and P-2 are two direct formulations of the "necessity" condition, the first one from a global point of view, the second from a local point of view. P-3 shows that this condition naturally comes to the notion of special groups of sup-generators 
that give access to a unique h-component through an h-opening. From the image processing point of view, this means that every h-component of an image can be selected individually with a simple opening.

Proposition 5. The h-connection $\left(\mathcal{C}_{m}^{+}, \bowtie_{m}\right)$ of functions with a unique maximum is accessible.

Proof. We show that $\mathcal{C}_{m}^{+}$verifies the property P-3. Let $a \in \mathcal{L}^{E}, h \in \gamma^{*}(a)$ and $x \in E$ such that $h(x)=\max _{y \in E} h(y)(h(x)$ is in the maximum of $h)$. Let the pulse $\delta_{x, h(x)}$ and we show that $\gamma_{\delta_{x, h(x)}^{*}}^{*}(a)=\{h\}$.

The inclusion $\{h\} \subseteq \gamma_{\delta_{x, h(x)}^{*}}^{*}(a)$ is direct as $\delta_{x, h(x)} \leq h$ and $h \in \gamma^{*}(a)$.

We now show the inverse inclusion $\gamma_{\delta_{x, h(x)}^{*}}^{*}(a) \subseteq\{h\}$. Let $h^{\prime} \in \gamma_{\delta_{x, h(x)}^{*}}^{*}(a)$. We start by showing that $\bowtie_{m}\left(\left\{h, h^{\prime}\right\}\right)$ is true. Let $t \in \mathcal{L}$, we have: if $t \leq h(x)$ then $\vec{h}^{t} \neq \emptyset$ and $\vec{h}^{t^{\prime}} \neq \emptyset\left(\right.$ as $\left.\delta_{x, h(x)} \leq h^{\prime}\right)$, else if $t>h(x)$ then $\vec{h}^{t}=\emptyset(h(x)$ is the maximal height of $h)$. And yet $\forall t \in \mathcal{L}, t \leq h(x)$, we have $x \in h^{t} \cap h^{\top t} \neq$ $\emptyset$. So $\bowtie_{m}\left(\left\{h, h^{\prime}\right\}\right)$ is true. In consequence, $\bigvee\left\{h, h^{\prime}\right\} \in \mathcal{C}_{m}^{+}$, but $h, h^{\prime} \leq a$ so $\bigvee\left\{h, h^{\prime}\right\} \leq a$, and as $h$ and $h^{\prime}$ are h-connected components of $a$, we have either $h=h^{\prime}$ or $h$ and $h^{\prime}$ are not comparable. Assume that $h$ and $h^{\prime}$ are not comparable, then $h<\bigvee\left\{h, h^{\prime}\right\}, h^{\prime}<\bigvee\left\{h, h^{\prime}\right\}$, and as $\bigvee\left\{h, h^{\prime}\right\} \leq a, h$ and $h^{\prime}$ cannot be h-connected components of $a$. Thus we have $h=h^{\prime}$ and $\gamma_{\delta_{x, h(x)}^{*}}^{*}(a) \subseteq\{h\}$.

Thus, we have the double inclusion and $\gamma_{\delta_{x, h(x)}}^{*}(a)=\{h\}$. Every h-connected component of $a$ can be obtained by an h-opening. The h-connection $\mathcal{C}_{m}^{+}$verifies property $\mathrm{P}-3$ and is accessible.

Proposition 6. The h-connection $\left(\mathcal{C}_{p}^{+}, \bowtie_{p}\right)$ of flat functions is accessible.

Proof. The demonstration is similar to the previous one using the opening $\gamma_{\delta_{x, h(x)}^{*}}^{*}(a)=\{h\}$ with $x \in E$ such that $h(x)=a(y)$.

Accessible h-connections also have stronger properties:

Proposition 7. If $\mathcal{C}^{+}$is accessible, being given a family of h-components, the $h$-components of its supremum is the same family: $\forall\left\{h_{i}\right\} \subseteq \gamma^{*}(a),\left\{h_{i}\right\}=$ $\gamma^{*}\left(\bigvee\left\{h_{i}\right\}\right)$

Proof. We already have the first inclusion $\left\{h_{i}\right\} \subseteq \gamma^{*}\left(\bigvee\left\{h_{i}\right\}\right)$ by proposition 2 . The second inclusion $\gamma^{*}\left(\bigvee\left\{h_{i}\right\}\right) \subseteq\left\{h_{i}\right\}$ is directly given by P-2: let $h \in$ $\gamma^{*}\left(\bigvee\left\{h_{i}\right\}\right)$, as we have $h \leq \bigvee\left\{h_{i}\right\}, \mathrm{P}-2$ says that $h$ belongs to $\left\{h_{i}\right\}$. Finally, the double inclusion proves that $\left\{h_{i}\right\}=\gamma^{*}\left(\bigvee\left\{h_{i}\right\}\right)$.

Compared to proposition 2, this version is harder and it ensures that by selecting a family of h-components, its reconstruction by the supremum operator will not introduce new h-components. From the image processing point of view, this ensures that, when performing an h-connected filtering, a deleted h-component cannot re-appear after the reconstruction of the selected h-components. Another interesting property of accessible h-connections concerns z-operators: 
Proposition 8. If $\mathcal{C}^{+}$is accessible, the equivalence relations $\stackrel{a}{\sim}$ and $\stackrel{a_{*}}{\sim}$ are equivalent: $\forall s_{1}, s_{2} \in \mathcal{S},\left(s_{1} \stackrel{a}{\sim} s_{2}\right) \Leftrightarrow\left(s_{1} \stackrel{a_{*}^{*}}{\sim} s_{2}\right)$.

Proof. First, we show that $\forall s_{1}, s_{2} \in \mathcal{S}, s_{1} \stackrel{a_{*}}{\sim} s_{2} \Rightarrow s_{1} \stackrel{a}{\sim} s_{2}$, this part does not need the accessibility property. We have:

$$
\begin{aligned}
s_{1} \stackrel{a_{*}^{*}}{\sim} s_{2} \Leftrightarrow \gamma_{s_{1}}^{*}(a)=\gamma_{s_{2}}^{*}(a) \Rightarrow \bigvee \gamma_{s_{1}}^{*}(a)=\bigvee \gamma_{s_{2}}^{*}(a) & \Leftrightarrow \gamma_{s_{1}}(a)=\gamma_{s_{2}}(a) \\
& \Leftrightarrow s_{1} \stackrel{a}{\sim} s_{2}
\end{aligned}
$$

The reverse implication is given by: assume that we have $s_{1} \stackrel{a}{\sim} s_{2}$, which is equivalent to $\gamma_{s_{1}}(a)=\gamma_{s_{2}}(a)$. Let $h \in \gamma_{s_{1}}^{*}(a)$, we have $h \leq \gamma_{s_{1}}(a)=\bigvee \gamma_{s_{1}}^{*}(a)=$ $\bigvee \gamma_{s_{2}}^{*}(a)$. Then, P-2 implies that $h \in \gamma_{s_{2}}^{*}(a)$ and thus $\gamma_{s_{1}}^{*}(a) \subseteq \gamma_{s_{2}}^{*}(a)$. The same argument is used to prove that $\gamma_{s_{2}}^{*}(a) \subseteq \gamma_{s_{1}}^{*}(a)$ showing that $\gamma_{s_{2}}^{*}(a)=\gamma_{s_{1}}^{*}(a)$ which is equivalent to $s_{1} \stackrel{a}{\sim} s_{2}$.

This property removes the necessity of operating a choice between the two distinct approaches when dealing with z-operators. Thus it simplifies the process of creating image filters based on z-zones.

\section{Conclusion}

We have proposed an evolution of the notion of h-connection motivated by the desirable property that the decomposition of an image into connected components should be necessary and sufficient to describe this image. Due to this evolution, based on three equivalent axioms, we have set out several new important properties for image processing which ensure the consistency and simplify the use of h-connected filters. This theoretical work offers a strong basis for the development of hierarchical representations based on h-connections [9].

\section{References}

1. Serra, J.: Connectivity on complete lattices. JMIV 9(3) (1998) 231-251

2. Braga-Neto, U., Goutsias, J.: A theoretical tour of connectivity in image processing and analysis. JMIV 19(1) (2003) 5-31

3. Wilkinson, M.: An axiomatic approach to hyperconnectivity. In: ISMM. (2009) 35-46

4. Serra, J.: Image Analysis and Mathematical Morphology. II: Theoretical Advances. Academic Press, London, UK (1988)

5. Ouzounis, G.: Generalized Connected Morphological Operators for Robust Shape Extraction. PhD thesis, University of Groningen (2009)

6. Ouzounis, G., Wilkinson, M.: Hyperconnected attribute filters based on k-flat zones. IEEE TPAMI accepted (2010)

7. Nempont, O., Atif, J., Angelini, E., Bloch, I.: A new fuzzy connectivity measure for fuzzy sets. JMIV 34(2) (2009) 107-136

8. Wilkinson, M.: Hyperconnectivity, attribute-space connectivity and path openings: Theoretical relationships. In: ISMM. (2009) 47-58

9. Perret, B., Lefèvre, S., Collet, C.: Hyperconnections and hierarchical representations for grayscale and multiband image processing. IEEE TIP Submitted (2011) 